\title{
NUMERICAL SIMULATION OF ENTROPY GENERATION FOR CASSON FLUID FLOW THROUGH PERMEABLE WALLS AND CONVECTIVE HEATING WITH THERMAL RADIATION EFFECT
}

\author{
Obalalu Adebowale Martins, ${ }^{1, a, *}$, Kazeem Issa ${ }^{1, b}$, Abdulrazaq Abdulraheem ${ }^{1, c}$, Ajala \\ Olusegun Adebayo ${ }^{2, d}$, Adeosun Adeshina Taofeeq, ${ }^{3, e}$, Oluwaseyi Aliu ${ }^{4, f}$, Adebayo Lawal \\ Lanre $^{4, g}$, Wahaab Adisa Fatai ${ }^{5, h}$
}

${ }^{1}$ Department of Statistics and Mathematical Sciences, Kwara State University, Malete, Kwara State, Nigeria

E-mail: adebowale.obalalu17@kwasu.edu.ng ${ }^{\mathrm{a}}$, issa.kazeem@kwasu.edu.ng ${ }^{\mathrm{b}}$, abdulrazaq.abulraheem@kwasu.edu.ng ${ }^{\mathrm{c}}$

${ }^{2}$ Department of Pure and Applied Mathematics, Ladoke Akintola University of Technology, Ogbomoso, Nigeria

E-mail: oaajala@lautech.edu.ng ${ }^{\mathrm{d}}$

${ }^{3}$ Department of Mathematics University of Ilorin, Ilorin, Nigeria

E-mail: shite91@gmail.com ${ }^{\mathrm{e}}$

${ }^{4}$ Fundamental and Applied Science Department, Universiti Teknologi PETRONAS, Bandar Seri Iskandar, Malaysia ${ }^{5}$ Department of Physics, University of South Florida, Tampa, FL 33620-5700, USA

E-mail: oluwaseyialiu@gmail.com ${ }^{\mathrm{f}}$, adebayolawallanre@yahoo.com ${ }^{\mathrm{g}}$, fatai.wahaab@gmail.com ${ }^{\mathrm{h}}$

*corresponding author

\begin{abstract}
In this work, the influence of entropy generation analysis for an electrically conducting Casson fluid flow with convective boundary conditions has been numerically studied. The governing equations are analyzed numerically using weighted residual methods. Subsequently, the residuals were minimized using two different approaches of weighted residual method namely collocation weighted residual method (CWRM) and Galerkin weighted residual method (GWRM) and computed numerically using MATHEMATICAL software. The impacts of governing parameters on Casson flow velocity, temperature profile, local skin friction, and Nusselt number were analysed. The obtained solutions were used to determine the heat transfer irreversibility and bejan number of the model. The results of the computation show that the effect of thermophysical properties such as thermal radiation parameter, suction/injection parameter, magnetic field parameter, radiation parameter, and Eckert number has a significant influence on Skin friction coefficient $\left(C_{f}\right)$ and local Nusselt number $\left(N_{u}\right)$ when compared to the Newtonian fluid. The findings from this study are relevant to advances in viscoelasticity and enhanced oil recovery.
\end{abstract}

Keywords: Thermal radiation, permeable walls, collocation method, Casson liquid, entropy generation. 


\section{Introduction}

Casson fluid (CF) has received significant attention in numerous branches of many areas of science, engineering, and food processing (Gireesha et al. 2019). Typical examples of common commodities exhibiting CF properties include oil, honey, jelly, and paints. CF model was first established by Casson (1959). The study establishes the prediction flow of pigment-oil suspension. Mukkopadhyay et al. (2013) reported CF flow of hydromagnetic and thermal convection heat transfer in a stretched permeable surface. The authors affirmed that the enhancement in non-Newtonian Casson parameter reduces the yield stress of the fluid flow and increases the fluid temperature. Hari and Harshad (2016) have studied Hydromagnetic Radiative CF flow through a vertical plate entrenched in a porous medium. Their results exhibited that the Casson parameter increases the fluid flow and decreases the temperature profile. Shaw et al. (2016) reported a numerical simulation of CF flow of Non-linear thermal convection with convective boundary conditions. The authors demonstrated that the velocity profile increases with rheological parameter. Ibukun et al. (2016) showed that the Newtonian fluid model reduced to non-Newtonian fluid especially when the wall stress is higher than the yield stress. Recently, Pop and Sheenet (2017) presented the thermal radiation effect on non-Newtonian CF in an enclosed space. The authors concluded that the rheological parameter enhances the fluid temperature. Gbadeyan et al. (2020) employed a numerical method Galerkin method to investigate the hydromagnetic flow of a $\mathrm{CF}$ in the existence of non-Darcian porous medium with variable thermal conductivity and viscosity effect. Their outcomes showed that the rheological parameter enhanced both the velocity and temperature profile.

Recently, the study of radiative effect has become important due to the widespread in scientific and environmental processes such as heating, solar power technology, and electrical power generation where more details can be found in a review by Salmeen et al. (2014). Moreover, Turkyilmazogylu et al. (2011) studied the effect of thermal radiation on unsteady hydromagnetic permeable walls with variable viscosity. Their results revealed that the thermal radiation parameter increased the temperature profile. Salmeen et al. (2014) discusses the thermal radiation effect of viscous incompressible fluid flow with convective heating. Their results showed that the radiative parameter increases the temperature profile. Kalida (2011) presented a hydromagnetic boundary flow over a moving surface flow in a saturated porous medium. Also, Anhter et al. (2017) discussed the effect of thermal radiation on Mhd natural convection boundary layer flow. Modal et al. (2015) considered the influence of thermal radiation on MHD asymmetric stagnantpoint flow

All the above studies are quite limited to the first law analysis of thermodynamics. Whereas, the second law has been utilized in different flow problems as well as the thermal system. The work of Bejan (1982) is the fundamental study of entropy generation in fluid temperature. The study established the importance of temperature for entropy production through convectional heat transfer. Abolbashari et al. (2015) presented a numerical investigation of entropy generation CF flow in a stretching surface. It was observed that the non-Newtonian Casson parameter boosts the entropy Generation number. Aziz and Makinde (2010) studied a rectangular enclosure of CF with thermal radiation effect and chemical reaction. They reported that the entropy production intensifies while increasing the rheological parameter. Recently, El-Aziz and Afify (2019) presented a second law analysis of MHD CF flow on a Stretching Sheet with a velocity slip. Their results revealed that the Casson parameter increases the heat transfer wall. Besides, Adesanya et al. (2017) studied the possibility of entropy reduction generated in a viscous convection cooling system. Ajala et al. (2019) investigate the impact of heat generation on forced convective flow through the permeable wall using a weighted residual collocation method. Razaq and Aregbesola (2012) discussed the weighted residual method in a semi-domain using un-partitioned methods. Eegunjobi and Makinde (2013) studied the second law thermodynamic of a variable viscous 
MHD channel flow with suction/injection. However, this study does not consider CF flow. In another study, Ananthaswamy et al. (2020) studied second law analysis in a variable viscous MHD channel flow with numerical Investigation. Again, the thermal radiation effect, entropy generation, and skin friction coefficient together with local Nusselt number were neglected.

In this study, the Casson rheological model on a steady, incompressible flow of an electrically conducting viscosity fluid, between two fixed permeable walls with thermal radiation effect and convective boundary condition has been studied. The present work used two different approaches of weighted residual method namely collocation method (CWRM) and Galerkin method (GWRM) to solve the governing equations. The distribution of fluid flow, temperature field, Bejan number, and irreversibility rate were evaluated via the thermodynamics second law. The structure of the methods is new and can be applied in developing models for blood oxygenators and hemodialyzers. Results show that the effect of thermophysical properties such as an increase in magnetic field parameter, suction/injection parameter, radiation parameter, Eckert number on skin friction coefficient $\left(C_{f}\right)$, and local Nusselt number $\left(N_{u}\right)$ have a significant influence when compared to the Newtonian fluid.

\section{Mathematical Analysis}

An electrically viscous incompressible fluid bounded by two permeable parallel plates with Casson liquid has been considered. As can be seen in Fig. 1, a constant Magnetic field of strength $B_{o}$ is imposed transversely in the y-direction. The applied magnetic field was assumed to be strong enough so that the induced magnetic field is weak. The top and bottom wall temperature are non-uniform under radiative heat transfer. With this assumption, the upper and lower plates are convective heated when the injection and suction occur, respectively.

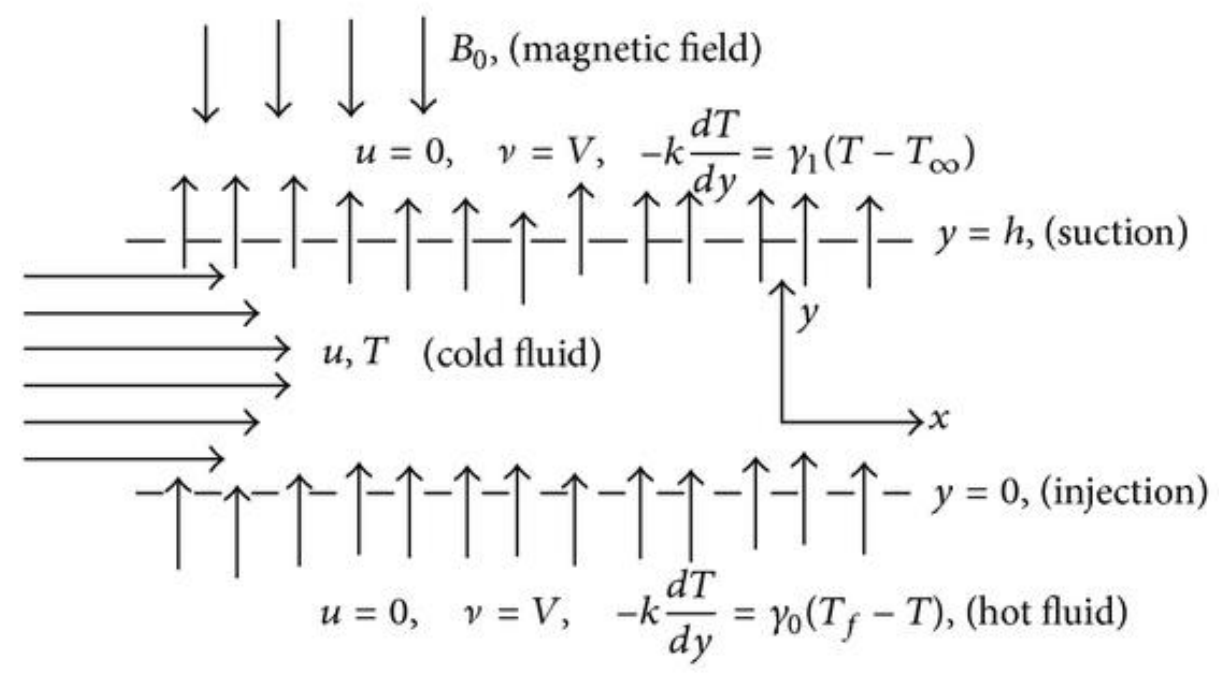

Fig. 1. Schematic depiction of the problem (Egunjobi and Makinde 2013)

The rheological equation of an isotropic and incompressible flow of CF can be written as equation (1) (El-Aziz and Afify 2019) 


$$
\left.\begin{array}{c}
\tau_{i j}=2\left(\mu_{B}+\frac{P_{y}}{\sqrt{2 \pi}}\right) e_{i j} \text { when } \pi>\pi_{c} \\
\tau_{i j}=2\left(\mu_{B}+\frac{P_{y}}{\sqrt{2 \pi c}}\right) e_{i j} \text { when } \pi<\pi_{c}
\end{array}\right\}
$$

$p_{y}$ is the fluid yield stress of liquid and can expressed Mathematically as

$$
P_{y}=\frac{\mu_{B} \sqrt{2 \pi}}{\beta}
$$

Where

$$
\mu_{f}=\mu_{B}+\frac{P_{y}}{\sqrt{2 \pi}}
$$

by substituting equation (2) into equation (3), the kinematic viscosity can be obtained as

$$
v_{f}=\frac{\mu_{B}}{\rho_{f}}\left(1+\frac{1}{\beta}\right)
$$

$\beta$ is the Casson parameter and the modified governing equations (Akhter et al. 2017) are

$$
\begin{gathered}
V \frac{d u}{d y}=-\frac{1}{\rho} \frac{d P}{d x}+\left(1+\frac{1}{\beta}\right) \frac{1}{\rho} \frac{d}{d y}\left(\mu_{B}(T) \frac{d u}{d y}\right)-\frac{\sigma B_{0}^{2} u}{\rho} \\
V \frac{d T}{d y}=\alpha \frac{d^{2} T}{d y^{2}}+\left(1+\frac{1}{\beta}\right) \frac{\mu_{B}(T)}{p c_{p}}\left(\frac{d u}{d y}\right)^{2}+\frac{Q_{0}}{p c_{p}}\left(T-T_{f}\right)-\frac{1}{p c_{p}} \frac{d q_{r}}{d y}+\frac{\sigma \mathrm{B}_{0}^{2} u^{2}}{p c_{p}} \\
E_{G}=\frac{K}{T_{\infty}^{2}}\left(\frac{d T}{d y}\right)^{2}+\left(1+\frac{1}{\beta}\right) \frac{\mu}{T_{\infty}^{2}}\left(\frac{d u}{d y}\right)^{2}+\frac{16 \sigma T_{1}^{3}}{3 K^{*}}\left(\frac{d^{2} T}{d y}\right)^{2}+\frac{\sigma B_{0}^{2} u^{2}}{T_{\infty}^{2}}
\end{gathered}
$$

with boundary conditions;

$$
\begin{aligned}
u(0) \quad=0, u(h)= & 0, \\
-k \frac{d T}{d y}(0) \quad & =\gamma_{0}\left(T_{f}-T(0)\right),-k \frac{d T}{d y}(0)=\gamma_{1}\left(T(h)-T_{\infty}\right)
\end{aligned}
$$

using Roseland approximations, the radiative heat flux is giving by $q_{r}=-\frac{4 \sigma}{3 k^{*}} \frac{d T^{4}}{d y}$ where $\sigma_{1}$ is the Stefan-Boltzman constant and $k_{1}^{*}$ is the mean absorption coefficient for thermal radiation. The variable plastic dynamic viscosity for non-newtonian fluid can be expressed as

$$
\mu_{B}(T)=\mu^{*}{ }_{B} \ell^{-a^{*}\left(T-T_{\infty}\right)}
$$

where $\mu_{B}^{*}$ is the constant value of the coefficient of viscosity, $a^{*}$ and $\mathrm{b}$ is empirical constants, The following non-dimensional quantities were introduced: 


$$
\begin{aligned}
& G=-\frac{\partial \bar{p}}{\partial x}, \mu=\frac{\bar{\mu}}{\mu_{0}}, E_{C}=\frac{v^{2}}{c_{p}}\left(T_{f}-T_{\infty}\right), \\
& \delta=\frac{Q_{0}}{k}, W=\frac{u}{v}, X=\frac{x}{h}, \alpha=\frac{k}{p c_{p}}, \Omega=\frac{T_{f}-T_{\infty}}{T_{\infty}}, \\
& \theta=\frac{T-T_{\infty}}{T_{f}-T_{\infty}}, \eta=\frac{v}{h}, M=\frac{\sigma B_{0}^{2} H^{2}}{\mu u}, \varepsilon=a^{*}\left(T_{f}-T_{\infty}\right), \\
& B i_{0}=\frac{\gamma_{0} h}{k}, B i_{1}=\frac{\gamma_{1} h}{k}, R=\frac{k K^{*}}{4 \sigma T_{1}^{3}}, S=\frac{v h}{v}, \\
& \bar{P}=\frac{P h}{\mu_{0} V}, \tau_{w}=\left(\mu_{B}+\frac{P_{y}}{\sqrt{2 \pi}}\right) \mu_{B}(T) \frac{d u}{d y},
\end{aligned}
$$

In equations (1) to (10), we obtained the following dimensionless equations:

$$
\begin{gathered}
\left(1+\frac{1}{\beta}\right) \frac{d^{2} w}{d \eta^{2}}-\left(1+\frac{1}{\beta}\right) \epsilon \frac{d \theta}{d \eta} \frac{d w}{d \eta}-\ell^{\epsilon \theta}\left(S \frac{d w}{d \eta}+M w-G\right)=0 \\
\left(1+\frac{1}{\beta}\right) \frac{d^{2} w}{d \eta^{2}}-\left(1+\frac{1}{\beta}\right) \epsilon \frac{d \theta}{d \eta} \frac{d w}{d \eta}-\ell^{\epsilon \theta}\left(S \frac{d w}{d \eta}+M w-G\right)=0 \\
N_{s}=\frac{T_{0}^{2} h^{2} E_{G}}{k\left(T_{f}-T_{\infty}\right)^{2}}=\left(1+\frac{4}{3} R\right)\left(\frac{d \theta}{d \eta}\right)^{2}+\frac{B r}{\Omega}\left[\left(1+\frac{1}{\beta}\right) \ell^{-\epsilon \theta}\left(\frac{d w}{d \eta}\right)^{2}+H a w^{2}\right]
\end{gathered}
$$

The corresponding initial and boundary condition are:

$$
\begin{gathered}
w(0)=0 \text { and } w(1)=0 \\
\frac{d \theta}{d \eta}(0)=B i_{0}(\theta(0)-1) \text { and } \frac{d \theta}{d \eta}(0)=B i_{0}(1)
\end{gathered}
$$

Other quantities of concern are the skin friction coefficient, nusselt number and bejan number which can be given as

$$
C_{f}=\frac{\tau_{w}^{h}}{\mu_{0} u}, N u=\frac{q_{w}^{h}}{k\left(T_{f}-T_{\infty}\right)}, B e=\frac{N_{1}}{N_{S}}=\frac{1}{1+\Phi}, \Phi=\frac{N_{2}}{N_{1}}
$$

Using the similarity and dimensionless variables in (1), we get

$$
\begin{gathered}
C_{f}=\left\{\left(1+\frac{1}{\beta}\right) \ell^{-\epsilon \theta} \frac{d w}{d \eta}\right\}_{\eta=0,1}, \quad N u=\left\{\left(1+\frac{4}{3} R\right)\left(\frac{d \theta}{d \eta}\right)\right\}_{\eta=0,1} \\
N_{1}=\left(1+\frac{4}{3} R\right)\left(\frac{d \theta}{d \eta}\right)^{2}, N_{2}=\frac{B r}{\Omega}\left[\left(1+\frac{1}{\beta}\right) \ell^{-\epsilon \theta}\left(\frac{d w}{d \eta}\right)^{2}+H a w^{2}\right]
\end{gathered}
$$

where $N_{1}$ represent the irreversibility outcome from heat transfer, $N_{2}$ represent entropy generation due to the viscous dissipation magnetic field (Obalalu et al. 2020), and $\Phi$ is described 
as the rate of entropy generated due to fluid friction. The heat irreversibility is controlled at $B e=1$ and $B e=0$ when viscous dissipation is the dominant contributor to entropy generation, whereas $B e=0.5$ is assigned to contribute equally.

\section{Method of Solution}

\subsection{The Application of collocation weighted residual}

To obtain solutions to governing equations (11) and (12) using the boundary conditions in equation (14) and (15), the collocation weighted residual method is used. Therefore, solutions are assumed for equations (11) and (12) as follows:

$$
w=\sum_{n=0}^{k} a_{k} \eta^{k}, \theta=\sum_{n=0}^{k} b_{k} \eta^{k}
$$

Substituting equation (15) into (10) into (13) to have residual functions

$$
\begin{aligned}
& \left.R_{w}(\eta)\right)=\left(1+\frac{1}{\beta}\right)\left(\begin{array}{c}
2 a_{2}+6 \eta a_{3}+12 \eta^{2} a_{4}+20 \eta^{3} a_{5} \\
+30 \eta^{4} a_{6}+42 \eta^{5} a_{7}+56 \eta^{6} a_{8}+72 \eta^{7} a_{9}+90 \eta^{8} a_{10}
\end{array}\right) \\
& -e^{\epsilon\left(b_{0}+\eta b_{1}+\eta^{2} b_{2}+\eta^{3} b_{3}+\eta^{4} b_{4}+\eta^{5} b_{5}+\eta^{6} b_{6}+\eta^{7} b_{7}+\eta^{8} b_{8}+\eta^{9} b_{9}+\eta^{10} b_{10}\right)} \\
& -G+R\left(a_{1}+2 \eta a_{2}+3 \eta^{2} a_{3}+4 \eta^{3} a_{4}+5 \eta^{4} a_{5}+6 \eta^{5} a_{6}+7 \eta^{6} a_{7}+8 \eta^{7} a_{8}+9 \eta^{8} a_{9}+10 \eta^{9} a_{10}\right) \\
& -\left(1+\frac{1}{\beta}\right) \epsilon\left(a_{1}+2 \eta a_{2}+3 \eta^{2} a_{3}+4 \eta^{3} a_{4}+5 \eta^{4} a_{5}+6 \eta^{5} a_{6}+7 \eta^{6} a_{7}+8 \eta^{7} a_{8}+9 \eta^{8} a_{9}+10 \eta^{9} a_{10}\right) \\
& \left(b_{1}+2 \eta b_{2}+3 \eta^{2} b_{3}+4 \eta^{3} b_{4}+5 \eta^{4} b_{5}+6 \eta^{5} b_{6}+7 \eta^{6} b_{7}+8 \eta^{7} b_{8}+9 \eta^{8} b_{9}+10 \eta^{9} b_{10}\right)
\end{aligned}
$$

and

$$
\begin{aligned}
& R_{\theta}(\eta)=e^{-\epsilon\left(b_{0}+\eta b_{1}+\eta^{2} b_{2}+\eta^{3} b_{3}+\eta^{4} b_{4}+\eta^{5} b_{5}+\eta^{6} b_{6}+\eta^{7} b_{7}+\eta^{8} b_{8}+\eta^{9} b_{9}+\eta^{10} b_{10}\right)} E c P r \\
& \left(1+\frac{1}{\beta}\right)\left(a_{1}+2 \eta a_{2}+3 \eta^{2} a_{3}+4 \eta^{3} a_{4}+5 \eta^{4} a_{5}+6 \eta^{5} a_{6}+7 \eta^{6} a_{7}+8 \eta^{7} a_{8}+9 \eta^{8} a_{9}+10 \eta^{9} a_{10}\right)^{2} \\
& +\operatorname{EcHPr}\left(a_{0}+\eta a_{1}+\eta^{2} a_{2}+\eta^{3} a_{3}+\eta^{4} a_{4}+\eta^{5} a_{5}+\eta^{6} a_{6}+\eta^{7} a_{7}+\eta^{8} a_{8}+\eta^{9} a_{9}+\eta^{10} a_{10}\right)^{2} \\
& +\left(1+\frac{4 R d}{3}\right)\left(2 b_{2}+6 \eta b_{3}+12 \eta^{2} b_{4}+20 \eta^{3} b_{5}+30 \eta^{4} b_{6}+42 \eta^{5} b_{7}+56 \eta^{6} b_{8}+72 \eta^{7} b_{9}+90 \eta^{8} b_{10}\right) \\
& -\operatorname{PrR}\left(b_{1}+2 \eta b_{2}+3 \eta^{2} b_{3}+4 \eta^{3} b_{4}+5 \eta^{4} b_{5}+6 \eta^{5} b_{6}+7 \eta^{6} b_{7}+8 \eta^{7} b_{8}+9 \eta^{8} b_{9}+10 \eta^{9} b_{10}\right)
\end{aligned}
$$

The collocation point method used in this work is defined as

$$
N j=a+\frac{b-a}{n} j, j=0,2, \ldots, n .
$$

With this method, a system of $2 n+2$ algebraic equation was obtained with a coefficient $a_{n}$ and $b_{n}$ 


\subsection{The Application of Galerkin weighted residual method}

By applying the Galerkin weighted residual method (GWRM), we assumed the trial function for $w(\eta)$ and $\theta(\eta)$ as follows (Razaq and Aregbesola 2020)

$$
w=\sum_{k=0}^{n} a_{k} * e^{\left(-x * \frac{k}{4}\right)} \text { and } \theta=\sum_{k=0}^{n} a_{k} * e^{\left(-x * \frac{k}{4}\right)}
$$

by substituting equation (11) and (12) into equation (23), to obtain

$$
\begin{aligned}
& R_{w}(\eta)=\left(1+\frac{1}{\beta}\right)\left(\begin{array}{c}
\frac{1}{16} x 4 a_{1}+\frac{1}{4}-x 2 a_{2}+\frac{9}{16} 3 x 4 a_{3}+e^{-x} a_{4}+\frac{25}{16} 5 x 4 a_{5}+\frac{9}{4} 3 \times 2 a_{6} \\
+\frac{49}{16} 7 x 4 a_{7}+4 e^{-2 x} a_{8}+\frac{81}{16} 9 x 4 a_{9}+\frac{25}{4} 5 x 2 a_{10}
\end{array}\right) \\
& \mathrm{e}^{\epsilon\left(\mathrm{e}^{-x / 4} b_{1}+\mathrm{e}^{-x / 2} b_{2}+\mathrm{e}^{-3 x / 4} b_{3}+\mathrm{e}^{-x} b_{4}+\mathrm{e}^{-5 x / 4} b_{5}+\mathrm{e}^{-3 x / 2} b_{6}\right)} \\
& \left(+H\left(a_{0}-x 4 a_{1}-3 x 4 a_{3}+e^{-x} a_{4}-5 x 4 a_{5}+3 x 2 a_{6}-7 x 4 a_{7}+e^{-2 x} a_{8}-9 x 4 a_{9}+5 \times 2 a\right)\right) \\
& -\left(1+\frac{1}{\beta}\right) \epsilon\left(\begin{array}{c}
-\frac{1}{4} x 4 a_{1}-\frac{1}{2} x 2 a_{2}-\frac{3}{4} 3 x 4 a_{3}-e^{-x} a_{4}-\frac{5}{4} 5 x 4 a_{5} \\
-\frac{3}{2} 3 x 2 a_{6}-\frac{7}{4} 7 x 4 a_{7}-2 e^{-2 x} a_{8}-\frac{9}{4} e-9 x 4 a_{9}-\frac{5}{2} 5 \times 2 a_{10}
\end{array}\right) \\
& \left(-\frac{1}{4} x 4 b_{1}-\frac{1}{2} \times 2 b_{2}-\frac{3}{4} 3 x 4 b_{3}-e^{-x} b_{4}-\frac{5}{4} 5 x 4 b_{5}-\frac{3}{2}-3 x 2 b_{6}\right)
\end{aligned}
$$

and

$$
\begin{aligned}
& R_{\theta}(\eta)=e^{-\epsilon\left(x 4 b_{1}+x 2 b_{2}+3 x 4 b_{3}+e^{-x} b_{4}+5 x 4 b_{5}+-3 x 2 b_{6}\right)} \operatorname{EcPr}\left(1+\frac{1}{\beta}\right) \\
& \left(\begin{array}{r}
-a_{1}-\frac{1}{2} \times 2 a_{2}-\frac{3}{4} 3 x 4 a_{3}-e^{-x} a_{4}-\frac{5}{4} 5 \times 4 a_{5}-\frac{3}{2} 3 \times 2 a_{6} \\
-\frac{7}{4} 7 x 4 a_{7}-2 e^{-2 x} a_{8}-\frac{9}{4} 9 x 4 a_{9}-\frac{5}{2} x a_{10}
\end{array}\right)^{2} \\
& +\operatorname{EcHPr}\left(\begin{array}{c}
a_{0}+x 4 a_{1}-x 2 a_{2}+3 x 4 a_{3}+e^{-x} a_{4}-5 x 4 a_{5} \\
+3 x 2 a_{6}-7 x 4 a_{7}+e^{-2 x} a_{8}-9 x 4 a_{9}+5 x 2 a_{10}
\end{array}\right)^{2} \\
& -\operatorname{PrR}\left(-\frac{1}{4} x 4 b_{1}-\frac{1}{2} \times 2 b_{2}-\frac{3}{4}-3 x 4 b_{3}-e^{-x} b_{4}-\frac{5}{4} 5 x 4 b_{5}-\frac{3}{2} 3 \times 2 b_{6}\right) \\
& +\left(1+\frac{4 R d}{3}\right)\left(\frac{1}{16}-x 4 b_{1}+\frac{1}{4}-x 2 b_{2}+\frac{9}{16}-3 x 4 b_{3}+e^{-x} b_{4}+\frac{25}{16}-5 x 4 b_{5}+\frac{9}{4} e-3 \times 2 b_{6}\right)
\end{aligned}
$$

Using the boundary conditions in the equation in (14) and (15) on the trial function, and Choosing $\mathrm{N}=10$, The boundary condition gratified automatically from the interval $[0,1]$. The solution is in residual function $=0,1 \ldots 6, \mathrm{k}=1,2 \ldots 6$. The residual error was minimized accurately by putting the integral of the residual function of the interval [0 to 1]. Then the weighted functions were implemented for $i=0,1, \ldots, N-2, k=1,2, \ldots, N-1$ to 0 , such that 


$$
\int_{0}^{1} R_{w} e^{-\frac{i \eta}{4}} d \eta=0 \text { and } \int_{0}^{1} R_{\theta} e^{-\frac{i \eta}{4}} d \eta=0
$$

Finally, MATHEMATICAL software was used to compute the unknown coefficients $\left(a_{i}\right.$ and $\left.b_{k}\right)$.

\section{Result and Discussion}

The numerical solutions to the formulated problems discussed in the previous sections are examined. Fig. 2 to Fig. 5 illustrate the effect of the Biot numbers, Brinkman-number, Suction/injection parameter, magnetic field parameter, Casson parameter, Radiation parameter, and Bejan number, heat generation parameter on the fluid flow $(w(\eta))$, fluid temperature $(\theta(\eta))$, entropy generation $(N s)$, and Bejan Number $(B e)$. The paramete $G=1, R=1, E c=1, S=0.5, \epsilon=0.5, M=0.2 \delta=1, \beta=1.5, B r \Omega^{-1}=0.5, \operatorname{Pr}=0.71, B i_{0,1}=0.1, \quad$ are default values used in this study and the parameters remain fixed throughout the research unless otherwise stated. The result presented in Table 1 confirms the accuracy of the exact solution as well as the collocation method and Galerkin method. Table 2 exhibit the comparison of current studies and previously published results. (Makinde and Eegunjobi 2013) and (Ananthaswamy et al. 2016 ) when: $R e=1, H a=1, G=1, \delta=R=\epsilon=0$ for Newtonian fluid $(\beta=\infty)$ an excellent agreement can be observed. Skin friction coefficient and Nusselt number. Table 3 and Table 4 display the non-Newtonian $\mathrm{CF}$ and Newtonian fluid $(\beta=\infty)$ for local Nusselt number and skin friction coefficient, with various values of $M, S, N$, and $E_{c}$ respectively. For both cases of nonNewtonian CF and Newtonian fluid $(\beta=\infty)$, it is found that the magnetic field parameter $(M)$ rises with the value of the Nusselt number $N u$. Whereas, the magnetic field parameter reduces the Skin friction coefficient in both cases. It was found that the magnetic field tends to oppose the damping force. From Table 3 and Table 4 the viscous dissipation in term of Eckert number enhances Skin friction coefficient and reduce the magnitude of Nusselt number $N u$. The values of $(\beta=\infty)$ the Skin friction coefficient and local Nusselt number increase gradually for rising values of suction/injection parameter for the both cases. In addition, we observed the suction/injection parameter moves smoothly towards the direction of the wall temperature Ajala et al (2019) presented in Table 3 and Table 4. Besides, both the magnitude of Skin friction coefficient and local Nusselt number values increses with incresing thermal radiation parameter. As expected, the thermal radiation increases gradually with the high temperature in the channel walls (Turkyilmazoglu 2011). Finally, the impact of Non-Newtonian CF on the Skin friction coefficient and local Nusselt number $N u$ are very noticeable when compared to the Newtonian fluid. CF acquires high shearing within the fluid channel as the case with blood models (Boyd et al. 2007). likewise, Casson liquid fluid enhances the heat transfer rate to generate vasodilation (Kataria 2016). 


\begin{tabular}{lccc}
\hline$\eta$ & CWRM $(W(\eta))$ & GWRM $(W(\eta))$ & exact solution $(W(\eta))$ \\
\hline 0 & 0 & 0 & 0 \\
0.1 & 0.03879297 & 0.03879297 & 0.03879297 \\
0.2 & 0.07114875 & 0.07114875 & 0.07114875 \\
0.3 & 0.09639032 & 0.09639032 & 0.09639032 \\
0.4 & 0.11376948 & 0.11376932 & 0.11376948 \\
0.5 & 0.12245933 & 0.12245933 & 0.12245933 \\
0.6 & 0.12154600 & 0.12154600 & 0.12154600 \\
0.7 & 0.11001953 & 0.11001953 & 0.11001953 \\
0.8 & 0.08676372 & 0.08676372 & 0.08676372 \\
0.9 & 0.05054498 & 0.05054498 & 0.05054498 \\
1.0 & 0 & 0 & 0 \\
\hline
\end{tabular}

Table 1. Comparison results between the collocation weighted residual method, Galerkin weighted residual method, and exact solution on CF velocity for $H=\delta=R=\epsilon=0$ for Newtonian fluid $(\beta=\infty) G=1, R e=1$.

\begin{tabular}{llll}
\hline$\eta$ & Present Study & \multicolumn{1}{c}{ Makinde and Eegunjobi, (2013) } & Ananthaswamy et al. (2016) \\
\hline 0 & \multicolumn{1}{c}{0} & \multicolumn{1}{c}{0} & 0 \\
0.1 & 0.035823 & 0.035822 & 0.035822 \\
0.2 & 0.065264 & 0.065264 & 0.065264 \\
0.3 & 0.087963 & 0.087963 & 0.087963 \\
0.4 & 0.103449 & 0.103449 & 0.103449 \\
0.5 & 0.111128 & 0.111127 & 0.111127 \\
0.6 & 0.110257 & 0.110257 & 0.110257 \\
0.7 & 0.099930 & 0.0999304 & 0.099930 \\
0.8 & 0.079043 & 0.079042 & 0.079042 \\
0.9 & 0.046264 & 0.046264 & 0.046264 \\
1.0 & 0 & 0 & 0 \\
\hline
\end{tabular}

Table 2. Results for the velocity profile as compared with previous works for $R e=1, H a=1, G=1, \delta=R=\epsilon=0$ for Newtonian fluid $(\beta=\infty)$ 


\begin{tabular}{llllll}
\hline $\mathrm{M}$ & $\mathrm{S}$ & $\mathrm{E}_{\mathrm{c}}$ & $\mathrm{R}$ & $\begin{array}{l}\text { Skin-friction } \\
\text { coefficient }\end{array}$ & Nusselt-number \\
\hline $\mathbf{4 . 0}$ & 0.6 & 0.3 & 0.2 & 0.481466 & 1.56967 \\
$\mathbf{5 . 0}$ & 0.6 & 0.3 & 0.2 & 0.531545 & 1.48331 \\
$\mathbf{6 . 0}$ & 0.6 & 0.3 & 0.2 & 0.559841 & 1.39887 \\
4.0 & $\mathbf{0 . 3}$ & 0.3 & 0.2 & 0.278461 & 1.54178 \\
4.0 & $\mathbf{0 . 9}$ & 0.3 & 0.2 & 0.669694 & 1.62189 \\
4.0 & $\mathbf{1 . 5}$ & 0.3 & 0.2 & 0.765255 & 1.69619 \\
4.0 & 0.6 & $\mathbf{0 . 0}$ & 0.2 & 0.490456 & 1.79518 \\
4.0 & 0.6 & $\mathbf{0 . 6}$ & 0.2 & 0.490456 & 0.99749 \\
4.0 & 0.6 & $\mathbf{1 . 5}$ & 0.2 & 0.490456 & 0.39923 \\
4.0 & 0.6 & 0.3 & $\mathbf{0 . 0}$ & 0.811848 & 0.94631 \\
4.0 & 0.6 & 0.3 & $\mathbf{0 . 5}$ & 0.236111 & 1.24429 \\
4.0 & 0.6 & 0.3 & $\mathbf{0 . 9}$ & 0.173479 & 1.69884 \\
\hline
\end{tabular}

Table 3. Computed Numerical values of Skin friction coefficient and Nusselt number when $R, E c, M, S, \epsilon=0.1, \operatorname{Pr}=0.71, G=1$ Casson liquid fluid=1.5

\begin{tabular}{llllll}
\hline $\mathrm{M}$ & $\mathrm{S}$ & $\mathrm{E}_{\mathrm{c}}$ & $\mathrm{R}$ & $\begin{array}{l}\text { Skin friction } \\
\text { coefficient } \mathrm{C}_{\mathrm{f}}\end{array}$ \\
\hline $\mathbf{4 . 0}$ & 0.6 & 0.3 & 0.2 & 0.355176 & 1.46888 \\
$\mathbf{5 . 0}$ & 0.6 & 0.3 & 0.2 & 0.389367 & 1.36241 \\
$\mathbf{6 . 0}$ & 0.6 & 0.3 & 0.2 & 0.416519 & 1.26479 \\
4.0 & $\mathbf{0 . 3}$ & 0.3 & 0.2 & 0.219918 & 1.43813 \\
4.0 & $\mathbf{0 . 9}$ & 0.3 & 0.2 & 0.459863 & 1.53328 \\
4.0 & $\mathbf{1 . 5}$ & 0.3 & 0.2 & 0.496965 & 1.61746 \\
4.0 & 0.6 & $\mathbf{0 . 0}$ & 0.2 & 0.355178 & 1.67722 \\
4.0 & 0.6 & $\mathbf{0 . 6}$ & 0.2 & 0.355178 & 0.95688 \\
4.0 & 0.6 & $\mathbf{1 . 5}$ & 0.2 & 0.355101 & 0.43331 \\
4.0 & 0.6 & 0.3 & $\mathbf{0 . 0}$ & 0.447734 & 0.96908 \\
4.0 & 0.6 & 0.3 & $\mathbf{0 . 5}$ & 0.231347 & 1.23333 \\
4.0 & 0.6 & 0.3 & $\mathbf{0 . 9}$ & 0.183491 & 1.56629 \\
\hline
\end{tabular}

Table 4. Numerical values of Skin friction coefficient $\mathrm{C}_{\mathrm{f}}$ and Nusselt number $\mathrm{Nu}$ for different values of: $M, S, R, E c, \epsilon=0.1, \operatorname{Pr}=0.71, G=1$ Newtonian Fluid $=\infty$ 
The range of lower plate and upper plate moving velocities on CF are shown in Fig. 2a. The figure shows the impact of the $\mathrm{CF}$ parameter on the velocity profile. It is found that an increase in $\mathrm{CF}$ parameter contributes to an improvement in the fluid flow. Logically, the Casson rheological was used as a fluid by plastic dynamic viscosity which has a powerful reaction on yield stress Shaw et al. (2016). However, as Casson rheological near infinity, the fluid flow increases extremely. From Fig. $2 b$ we observed that an increase in CF parameter elevates entropy production as a result of an increase with injection at the upper plate and decreasing suction at the lower plate. A reduction in Casson liquid parameter was observed within the channel with irreversibility due to the exchange of heat, which controls the fluid flow (Gbadeyan et al. 2020) as shown in Fig. 2c. It was found that an increase in radiation parameter enhances the absorbing rate $(\mathrm{k})$ which decreases the temperature profile. Consequently, the thermal radiation parameter describes exactly the related additional conduction to thermal radiation (Akhter et al. 2017) as displayed in Fig. 2d. Fig. 2e displayed the impact of thermal radiation parameter on the entropy generation. The entropy production rate at the upper plate increases and reduces at the lower plate with an increasing radiative parameter. The thermal radiation parameter accelerates the entropy generation, which causes an enhancement in the entropy production. Fig. $2 \mathrm{f}$ displayed the impact of the radiative parameter on Bejan Number. Both the upper and lower plates reduce with increasing radiative parameter. The presence of thermodynamics irreversibility dominates the heat transfer entropy production.
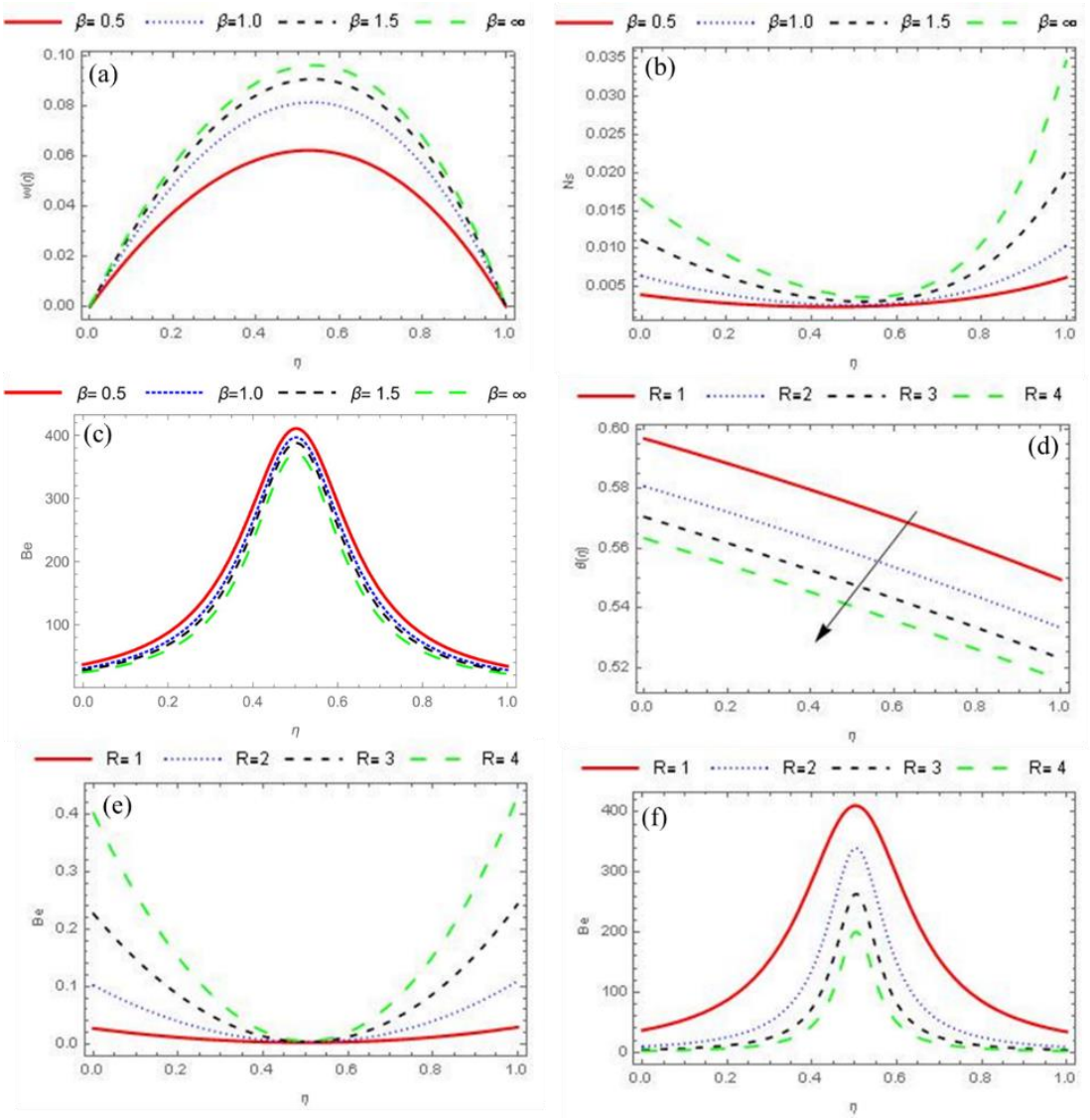

Fig. 2. Impact of (a, b, and c) CF parameter on fluid flow and entropy generation, Bejan number, $(\mathrm{d}, \mathrm{e}$, and $\mathrm{f})$, Thermal radiation parameter on the temperature profile, entropy generation, and Bejan number. 
Fig. 3a exhibit that the fluid flow slows down as the magnetic field parameter increases. This can be ascribed to the existence of Lorentz force which serves as a resisting force to the fluid flow. Hence, it slows down the fluid velocity, meanwhile its propensity to suppresses and decelerate the CF motion Obalalu et al.(2020). It can be seen from Fig. 3b that the impact of increasing magnetic field parameter is noticeably accelerated by the fluid temperature within the channel. Because the existence of ohmic heating acts as an additional source of heat to the fluid temperature. Fig. $3 c$ presents the impact of the magnetic field parameter on the entropy generation. The influence of magnetic field parameter on entropy generation weaker the upper plate, furthermore magnetic field parameter strongly increased the centerline region of the flow system. For the case of bejan number presented in Fig. 3d, it was found that the magnetic field parameter reduces the center region of the channel. In addition, bejan number strongly increased at the upper lower plate. Fig. 3e demonstrates the influence of the Suction/Injection parameter on Casson liquid flow and showed that the fluid velocity reduces and changes the direction against the upper plate. Whereas the Suction/Injection increases as the Casson liquid flow reduce due to injection at the lower plate, however, the rate of the fluid flow at the second plate strongly increases. Fig. 3f shows the influence of the Suction/Injection parameter on the fluid temperature. It can be observe that an increase in the Suction/Injection parameter increased the fluid temperature. It was observed that increasing Suction/Injection enhances the rate of entropy generation at the upper plate region.
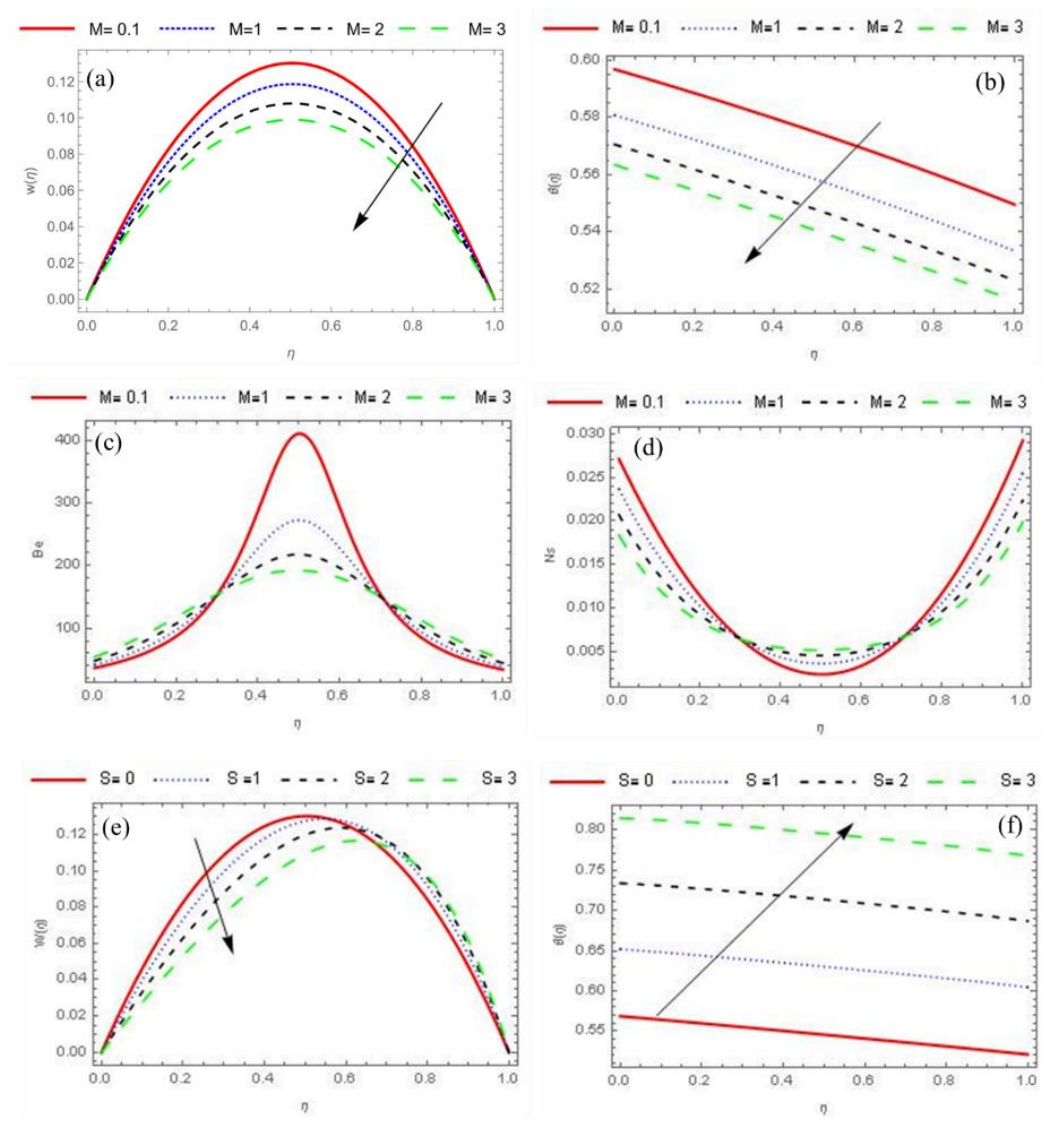

Fig. 3. Impact of (a, b, c, and d) magnetic field intensity on fluid flow, temperature profile, entropy generation, and Bejan number, (e, f) Suction/Injection parameter on fluid flow, temperature profile, entropy generation, and Bejan number. 
However, the entropy production rate decrease at the lower plate region as presents in Fig. 4a. Fig. $4 \mathrm{~b}$ illustrates that a similar response is observed for bejan Number with increasing Suction/Injection parameter. It is found that the Bejan number at lower permeable walls reduces, while a rise in the Bejan number occurs at the direction of the upper plate region. Meanwhile, as expected, the fluid injection control by irreversibility as a result of fluid friction, also the fluid suction wall region control by heat transfer irreversibility. Fig. 4c depicts the effect of convective heating $\left(B i_{0}\right)$ on fluid flow. It was found that as the convective heat rises, the CF flow at the lower plate increases. Whereas, the contrary is the case in Fig. $4 \mathrm{~d}$ where the $\left(B i_{0}\right)$ reduce the convective cooling heating at the upper plate. Fig. $4 \mathrm{e}$ depicts the impact of $\mathrm{Bi}_{0}$ on the fluid temperature. Increase in convective heat $\left(B i_{0}\right)$ enhanced the injection permeable wall and the contrary is the case in Fig. $4 \mathrm{f}$ where $\left(B i_{1}\right)$ reduce the convective heat loss at the suction permeable walls. 

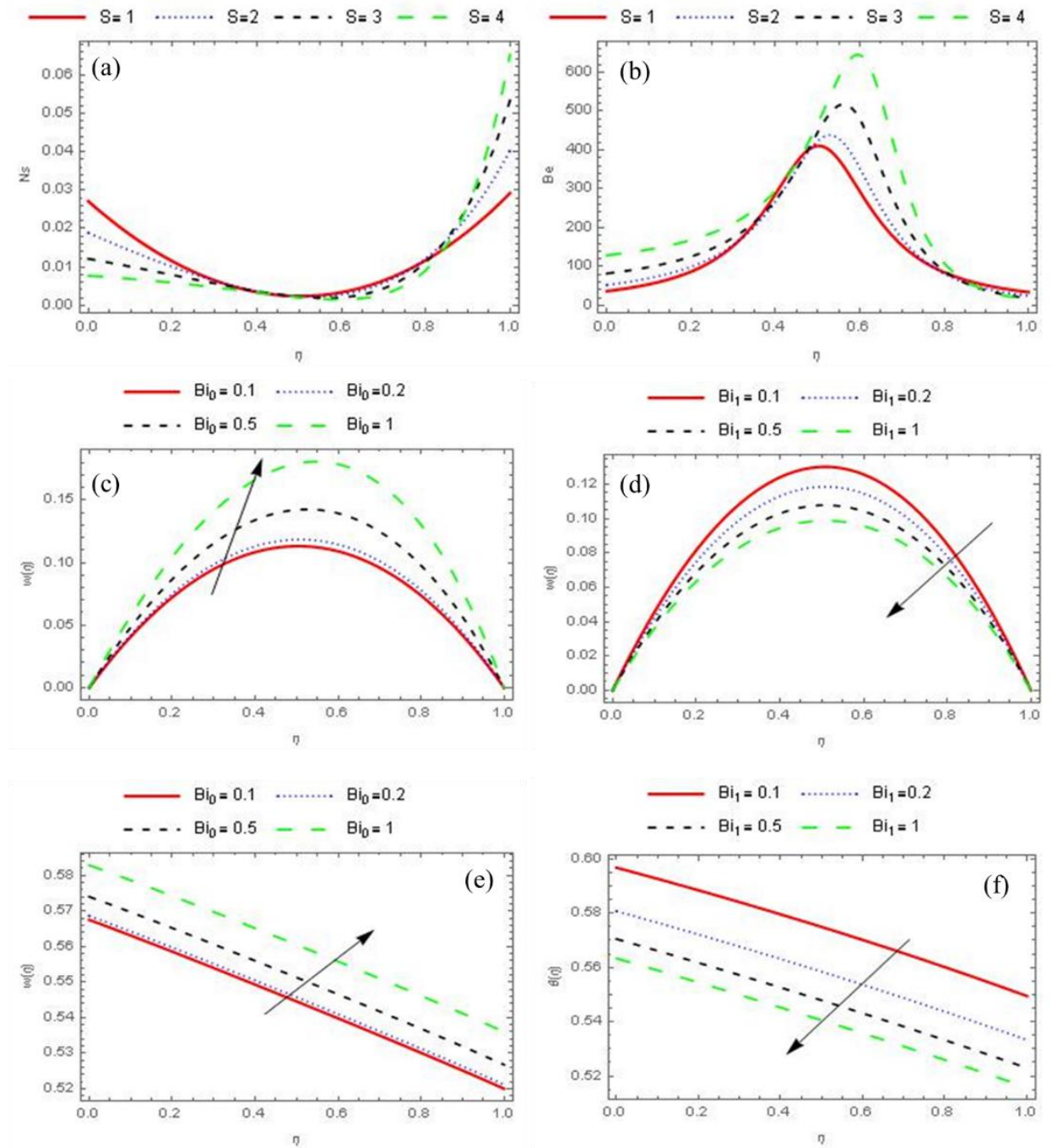

Fig. 4. Impact of (a and b) Suction/Injection parameter on entropy generation and Bejan number, $(c, d)$ Biot number on fluid flow and $(e, f)$ temperature profile

Fig. 5a illustrates the effect of the heat generation parameter on the fluid temperature. A rise in heat generation parameter enhances the fluid temperature, then the heat source generates an increment in heat transfer. Fig. $5 \mathrm{~b}$ presents the impact of the heat generation parameter on entropy production. A rise in entropy production is observed with increasing heat source, this is logical while the presence of the heat source contributes to the fluid friction irreversibility which eventually leads to cooling of the heated channel wall. Fig. $5 \mathrm{c}$ presents the effect of $\mathrm{B} \Omega^{-1}$ on entropy generation. The $B \Omega^{-1}$ displays an increasing and decreasing trend at the upper and lower plates, respectively, increasing the entropy generation in the channel-line. Fig. 5d displays the impact of $B \Omega^{-1}$ on Bejan number. It was observed that increasing the value of $B \Omega^{-1}$ enhances the fluid friction with irreversibility which does not affect the heat transfer irreversibility $\left(N_{1}\right)$. A higher value of $\Omega$ reduces the bejan number. Fig. 5e shows the graph of the residual functions $R(\eta)$. It was found that the residuals are minimized in the domain (0 to 1$)$. 

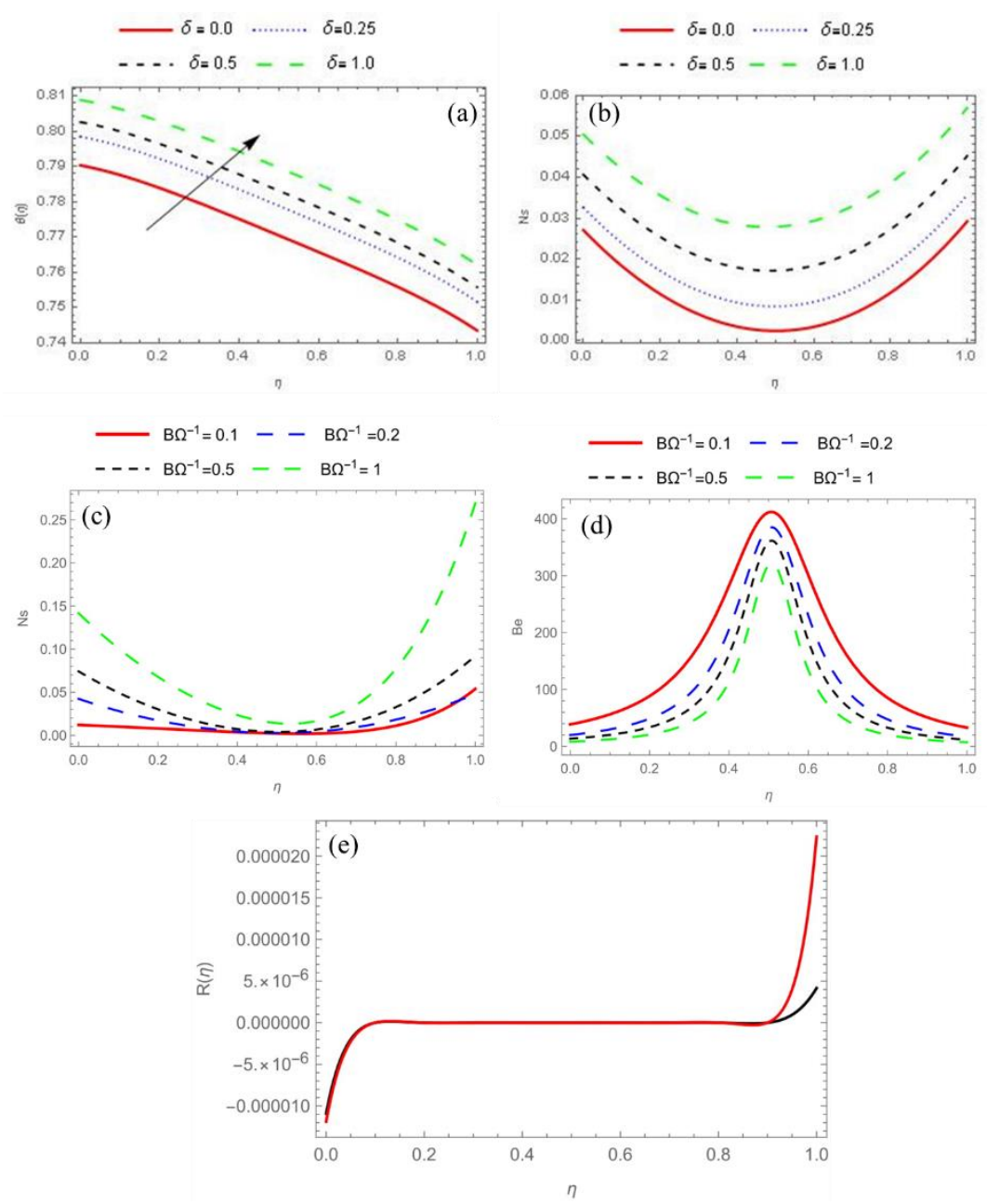

Fig. 5. Impact of $(a, b)$ heat generation parameter on temperature profile, and entropy generation, (c, d) Brinkman-number on entropy generation, Bejan number, (e) Minimized residual error $R(\eta)$.

\section{Conclusion}

In this work, we have successfully employed a Casson rheological model to analyze the second law thermodynamics for a steady, incompressible flow, electrically conducting viscosity fluid. A $\mathrm{CF}$ flow between two fixed permeable walls having thermal radiation effect and convective boundary condition was considered. The governing equations were solved using two different approaches of weighted residual method namely the Collocation method (CWRM) and Galerkin method (GWRM). The research has exhibits that the $\mathrm{CF}$ velocity reduces with increasing values of $S, M, R$, and $B i_{1}$, while it increases with increasing values of $\beta$ and $B i_{0}$. Similarly, the fluid temperature increases with higher values of $\mathrm{S}$ and $\mathrm{Bi}_{0}$, whereas it reduces with increasing values of $M$. Increasing values of $B \Omega^{-1}, \beta, \delta, \mathrm{R}$, and $\mathrm{S}$, enhance entropy production. The Bejan number decreases with increasing values of $\beta, M, R$, and $B \Omega^{-1}$, whereas an opposite trend was 
observed in the situation of $\mathrm{S}$. The result of $M, S, R$ and $E c$ on Skin friction coefficient $C_{f}$ and local Nusselt number $(\mathrm{Nu})$ is very noticeable when compared to the Newtonian fluid. Also, convective heating at the lower plate is more effective on the $\mathrm{CF}$ velocity than convective cooling at the upper plate. Hence the process of entropy generation is distinct towards the direction of permeable channel walls. The highlighted results above are significant in viscoelasticity and enhanced oil recovery.

\section{Acknowledgements}

The authors acknowledge the supports of staff of the department of statistics and mathematical sciences, Kwara State University.

Nomenclature

$\begin{array}{ll}x, y & \text { axial and normal coordinates } \\ A & \text { thermal diffusivity } \\ p & \text { fluid density } \\ t_{w} & \text { skin friction } \\ q_{w} & \text { heat flux } \\ u & \text { velocity of the fluid } \\ p & \text { fluid pressure } \\ \Sigma & \text { electrical conductivity } \\ B e & \text { Bejan number } \\ \operatorname{Re} & \text { Reynolds parameter } \\ K & \text { thermal conductivity coefficient } \\ B \Omega^{-1} & \text { Brinkman-number } \\ Y_{0} & \text { heat transfer coefficient of the lower plate } \\ B i_{0.1} & \text { Biot number } \\ C_{p} & \text { Specific heat at a constant pressure } \\ N & \text { radaition parameter } \\ T & \text { Channel fluid parameter } \\ \beta & \text { Cason parameter } \\ G & \text { pressure gradient parameter } \\ p r & \text { Pradndtl-number } \\ \operatorname{Re} & \text { Reynolds parameter } \\ t_{\infty} & \text { ambient temperature } \\ v & \text { uniform suction/injection velocity } \\ M & \text { magnetic field parameter } \\ \gamma_{1} & \text { heat transfer coefficient at the upper plate } \\ Q & \text { heat generation parameter } \\ & \end{array}$




$\begin{array}{ll}T_{h} & \text { Temperature of the hot fluid } \\ E_{c} & \text { Eckerts number }\end{array}$

\section{References}

Abolbashari M H, Freidoonimehr N, Nazari F, Rashidi M T (2015). Analytical modeling of entropy generation for Casson nano-fluid flow induced by a stretching surface, Advanced Powder Technology, 26, 542-552.

Adesanya S O, Falade J, Jangili S (2017). Irreversibility analysis for reactive third-grade fluid flow and heat transfer with convective wall cooling, Alexandria Engineering Journal, 56, 153-160.

Ajala OA, Obalalu A M, Abimbade S F, and Akinyemi O T (2019). Numerical Study of Forced Convective Heat Generation Flow through a Permeable Walls with Suction/Injection, International Journal of Scientific and Research Publications (IJSRP) 9, 336-348.

Akhter R, Mohammad M A, Babul H, and Musa M, (2017). Mhd free convection boundary layer flow over an inclined heated flat plate with thermal radiation, American Journal of Fluid Dynamics 44, 583-595.

Ananthaswamy V (2016). Mathematical expressions of entropy generation in a variable Viscosity MHD channel flow, mathematical expressions of entropy generation.

Aziz A, Makinde O D (2010). Analysis of entropy generation and thermal stability in slab, Journal of thermophysics and heat transfer, 24, 438-444.

Bejan A (1982). Second-law analysis in heat transfer and thermal design, Advances in heat transfer, 15, 1-58.

Boyd J, Buick M, Green S (2007). Analysis of the Casson and Carreau-Yasuda non-Newtonian blood models in steady and oscillatory flows using the lattice Boltzmann method, Physics of Fluids, 19, 9, p. 093103.

Casson N (1959). A flow equation for pigment-oil suspensions of the printing ink type, Rheology of disperse systems.

Egunjobi A, Makinde O D (2013). Entropy generation analysis in a variable viscosity MHD channel flow in with permeable walls and convective heating, Mathematical Problems in Engineering, 6,583-595.

El-Aziz M A, Afify A A (2019). MHD Casson fluid flow over a stretching sheet with entropy generation analysis and Hall influence, Entropy 21, 6, 592.

Gbadeyan J, Titiloye E. and Adeosun A. (2020). Effect of variable thermal conductivity and viscosity on Casson nanofluid flow with convective heating and velocity slip, Heliyon 6 , 037-076.

Gireesha B C, Srinivasa N, Shashikumar M, Macha J, Mahanthesh B (2019). Entropy generation and heat transport analysis of Casson fluid flow with viscous and Joule heating in inclined porous microchannel, Proceedings of the Institution of Mechanical Engineers, Part E: Journal of Process Mechanical Engineering, 233, 1173-1184

Kataria H R, Patel E. J (2016). Radiation and chemical reaction effects on MHD Casson fluid flow past an oscillating vertical plate embedded in porous medium, Alexandria Engineering Journal, vol. 55, no. 1,583-595,

Mansour M, El-Shaer N. M, (2001). Radiative effects on magnetohydrodynamic natural convection flows saturated in porous media, Journal of Magnetism Magnetic Materials, 237, 327-341,

Mondal S, Haroun N. A, Sibanda P, (2015). The effects of thermal radiation on an unsteady MHD axisymmetric stagnation-point flow over a shrinking sheet in presence of temperature dependent thermal conductivity with Navier slip, $P \operatorname{LoS} 9$, 553-685. 
Mukhopadhyay S, Vajravelu K, Van Gorder A. M. (2013). Casson fluid flow and heat transfer at an exponentially stretching permeable surface, Journal of Applied Mechanics and Technical Physics, 80, 344-786.

Obalalu A M, Wahaab F A, Adebayo L L (2020). Heat transfer in an unsteady vertical porous channel with injection/suction in the presence of heat generation, Journal of Taibah University for Science, 14, 541-548.

Oderinu R A, Aregbesola Y (2012). A Weighted Residual Method in a Semi-Infinite Domain Using Un-Partitioned Methods, International Journal of Applied Mathematics, 25, 25-31.

Oyelakan I. S, Mondal S, Sibanda P. J (2016). Unsteady Casson nanofluid flow over a stretching sheet with thermal radiation, convective and slip boundary conditions, Alexandria engineering journal, 55, 2, 1025-1035.

Pop I, Sheremet N (2017). Free convection in a square cavity filled with a Casson fluid under the effects of thermal radiation and viscous dissipation, International Journal of Numerical Methods for Heat Fluid Flow 25, 25-34.

Razaq A Aregbesola Y (2013). Weighted residual method in a semi-infinite domain using unpartitioned method, Int. J. Appl. Math, 25,25-34.

Shaw S, Mahanta G, Sibanda P J (2016). Non-linear thermal convection in a Casson fluid flow over a horizontal plate with convective boundary condition, Alexandria Engineering Journal, 55, 2, 1295-1304,

Turkyilmazoglu M. (2011). Thermal radiation effects on the time-dependent MHD permeable flow having variable viscosity, International Journal of Thermal Sciences, 50, 88-96.

Saleem M, Hossain M, Saha S C. Gu Y (2014). Heat transfer analysis of viscous incompressible fluid by combined natural convection and radiation in an open cavity, Mathematical Problems in Engineering.

Vyas P, Soni S (2016). Entropy analysis for MHD Casson fluid flow in a channel subjected to weakly temperature dependent convection coefficient and hydrodynamic slip, Rajasthan Academy of Physical Sciences, 15. 1-18. 